\title{
Raloxifene prevents stress granule dissolution, impairs translational control and promotes cell death during hypoxia in glioblastoma cells
}

\author{
Kathleen M. Attwood', Aaron Robichaud ${ }^{2,3}$, Lauren P. Westhaver ${ }^{4}$, Elizabeth L. Castle ${ }^{5}$, David M. Brandman ${ }^{3}$, \\ Aruna D. Balgi $i^{6}$, Michel Roberge ${ }^{6}$, Patricia Colp $\mathbb{1}^{4}$, Sidney Croul ${ }^{4}$, Inhwa Kim7, Craig McCormick $\mathbb{D}^{5}$, \\ Jennifer A. Corcoran ${ }^{8}$ and Adrienne Weeks ${ }^{1,2,3}$
}

\begin{abstract}
Glioblastoma (GBM) is the most common primary malignant brain tumor, and it has a uniformly poor prognosis. Hypoxia is a feature of the GBM microenvironment, and previous work has shown that cancer cells residing in hypoxic regions resist treatment. Hypoxia can trigger the formation of stress granules (SGs), sites of mRNA triage that promote cell survival. A screen of 1120 FDA-approved drugs identified 129 candidates that delayed the dissolution of hypoxiainduced SGs following a return to normoxia. Amongst these candidates, the selective estrogen receptor modulator (SERM) raloxifene delayed SG dissolution in a dose-dependent manner. SG dissolution typically occurs by 15 min posthypoxia, however pre-treatment of immortalized U251 and U3024 primary GBM cells with raloxifene prevented SG dissolution for up to $2 \mathrm{~h}$. During this raloxifene-induced delay in SG dissolution, translational silencing was sustained, elF2a remained phosphorylated and mTOR remained inactive. Despite its well-described role as a SERM, raloxifenemediated delay in SG dissolution was unaffected by co-administration of $\beta$-estradiol, nor did $\beta$-estradiol alone have any effect on SGs. Importantly, the combination of raloxifene and hypoxia resulted in increased numbers of late apoptotic/necrotic cells. Raloxifene and hypoxia also demonstrated a block in late autophagy similar to the known autophagy inhibitor chloroquine (CQ). Genetic disruption of the SG-nucleating proteins G3BP1 and G3BP2 revealed that G3BP1 is required to sustain the raloxifene-mediated delay in SG dissolution. Together, these findings indicate that modulating the stress response can be used to exploit the hypoxic niche of GBM tumors, causing cell death by disrupting pro-survival stress responses and control of protein synthesis.
\end{abstract}

\section{Introduction}

Glioblastoma multiforme (GBM) is the highest grade (IV) and most common primary brain tumor. GBM can occur de novo or from related lower-grade counterpart astrocytomas (Grade II/III); although all lower-grade astrocytomas will progress to GBM. Lower-grade astrocytomas are slow growing lesions characterized by the

\footnotetext{
Correspondence: Adrienne Weeks (a.weeks@dal.ca)

'Department of Surgery, Dalhousie University, Halifax, NS, Canada

${ }^{2}$ Department of Medical Neuroscience, Dalhousie University, Halifax, NS,

Canada

Full list of author information is available at the end of the article

Edited by I. Amelio
}

absence of both necrosis and vascular proliferation which are pathognomonic of GBM. The current treatment paradigm (surgery, radiation, and temozolomide) has a dismal 25\% 2-year survival rate ${ }^{1}$. Treatment failures occur at the primary site of occurrence, thus relapse fundamentally results from treatment resistance.

Tight regulation of protein synthesis is required to meet, but not exceed, the anabolic demands of the cell. In response to environmental stress, protein synthesis is rapidly arrested to prevent production of aberrant proteins and promote cell survival and restoration of homeostasis. There is strong evidence that this type of translation control plays an important role in cancer 
progression $^{2-5}$. Cancer cells place higher demands on the protein synthesis machinery, ramping up translation and subverting regulatory mechanisms. For example, GBM cells thrive in a stressful, low oxygen environment and survive radiation doses that would normally arrest protein synthesis and halt cell proliferation ${ }^{6}$. GBM is thought to recur because GBM cells can alter translational landscapes and survive hypoxic, radiated, and chemotherapeutic microenvironments ${ }^{7,8}$. Understanding mechanisms of translation control in GBM cells could enable the identification of new therapeutic targets.

Stress granules (SGs) are cytoplasmic, non-membranebound foci of mRNAs and RNA-binding proteins that form to spatially regulate mRNA stability, localization, and translation during the integrated stress response (ISR). SGs form in response to activation of at least one of the four stress-sensing sentinel kinases: protein kinase $R$ (PKR), PKR-like endoplasmic reticulum kinase (PERK), general control nonderepressible 2 (GCN2), and hemeregulated eukaryotic translation initiation factor 2 alpha kinase (HRI). These kinases phosphorylate eukaryotic translation initiation factor 2 alpha (eIF2 $\alpha$ ), which increases binding affinity between eIF $2 \alpha$ and the guanine nucleotide exchange factor eukaryotic translation initiation factor $2 \mathrm{~B}$ (eIF2B), thereby blocking the recharging of the eIF2-GTP-tRNA ${ }^{\text {iMet }}$ ternary complex and preventing translation initiation ${ }^{9,10}$. Bulk translationally stalled mRNAs are bound by aggregation-prone proteins with intrinsically disordered domains (e.g T-cell-restricted intracellular antigen-1 (TIA-1), TIA-1-related protein (TIAR), and Ras GTPase-activating protein-binding protein $1 / 2(\mathrm{G} 3 \mathrm{BP} 1 / 2))$ that drive SG aggregation ${ }^{11}$. However, this inhibition of bulk translation is incomplete, and SGs promote cell survival by re-prioritizing the translational apparatus to the generation of proteins that facilitate stress adaptation and cell survival. SGs also allow rapid resumption of protein synthesis following stress resolution, since mRNAs released from SGs are already bound to translation initiation machinery ${ }^{5}$.

SGs are highly dynamic structures that in physiologic conditions dissolve even in the persistence of stress ${ }^{12}$. A key step is the dephosphorylation of eIF2 $\alpha$, which recharges the eIF2-GTP-tRNA ${ }^{\text {Met }}$ ternary complex and allows re-entry in translation. Dephosphorylation can occur by the constitutively active reverter of eIF2 $\alpha$ phosphorylation $(\mathrm{CReP})$ or by the stress-induced phosphatase, growth arrest, and DNA damage-inducible protein $\left(\right.$ GADD34) ${ }^{13}$. However, timely dissolution of SGs requires functioning protein quality control (PQC). Severe stress increases the content of misfolded protein and defective ribosomal products (DRiPs). The aggregation of DRiPs and misfolded proteins into SGs results in SGs that are difficult to dissolve ${ }^{14,15}$. In instances where the stress overwhelms the PQC, SGs persist. Granulostasis would then affect the ability of cells to properly restore translation after stress, with potentially harmful effects and loss of cellular viability ${ }^{16}$.

Hypoxia is one of the canonical stressors that triggers SGs by activating PERK/PKR/GCN2 ${ }^{17-19}$. GBM, like many solid tumors, features extensive hypoxic regions. GBM tumor cells must have mechanisms to thrive despite the normally cytostatic/cytotoxic effects of hypoxia. The tightly regulated formation and dissolution of SGs is a key mechanism that allows cells to endure environmental stress. Therefore, we hypothesized that hypoxia-induced SGs play a significant role in GBM pathobiology. Importantly for cancer research, SGs can be pharmacologically manipulated, making SGs potential targets for therapy ${ }^{20-24}$. Utilizing high-throughput image analysis and the Prestwick Chemical Library, we screened 1120 compounds for the ability to disrupt SG dynamics. We discovered that selective estrogen receptor modulators (SERMs) including raloxifene prevent disassembly of hypoxia-induced SGs and promote cell death.

\section{Materials and methods \\ Cancer Genome Atlas interrogation}

The Cancer Genome Atlas (TCGA) was interrogated with GlioVis ${ }^{25}$, www.gliovis.bioinfo.cnio.es in February 2019 for PERK, GCN2, G3BP1, eIF2 $\alpha$, and GADD34 mRNA expression and survival in low-grade astrocytoma and GBM. Statistical analysis (Tukey's honest significant difference and log rank $p$-values) was determined using the statistical package included in the software. Corrections for multiple comparisons were made in both the expression data and Kaplan-Meier curve data.

\section{Immunohistochemistry}

A tissue microarray (TMA) consisting of 90 intact GBM cores from 45 patients and two normal cortex controls was obtained from Dr. Sidney Croul in the Department of Pathology at Dalhousie University. The TMA was serial sectioned and prepared for immunohistochemical labeling as previously described ${ }^{26}$. Tissue sections were labeled with primary antibodies: TIAR (BD Biosciences, 610352; diluted 1:60), G3BP2 (Sigma-Aldrich, HPA018304; diluted 1:1000), hematoxylin and eosin. Cytoplasmic staining was graded with the help of a pathologist. Cytoplasmic granular staining was graded from 0 to 4; 0 being no cytoplasmic granular staining, 1 being minority of cells with cytoplasmic granular staining, 2 being $\sim 50 \%$ of the cells with cytoplasmic granular staining, 3 being the majority of cells demonstrating cytoplasmic granular staining and 4 being all the cells (vascular endothelium excepted) exhibiting cytoplasmic granular staining.

\section{Cell lines and cell culture}

Immortalized human GBM U251 MG cells (a generous donation from J. Rutka; Sigma-Aldrich origin) and 
HEK293T cells (ATCC) were cultured in Dulbecco's modified Eagle's medium (DMEM) (high glucose, no sodium pyruvate) supplemented with $1 \%$ glutamine, penicillin-streptomycin, and $10 \%$ fetal bovine serum (Gibco). Primary U3024 MG (HGCC RRID:CVCL_IR67) cells were cultured on polyornithine and laminin-coated plates in 1:1 neurobasal and DMEM/F12 glutamax media supplemented with $1 \%$ penicillin-streptomycin, B-27, N-2 (Gibco), $10 \mathrm{ng} / \mathrm{mL}$ epidermal growth factor (EGF), and $10 \mathrm{ng} / \mathrm{mL}$ fibroblast growth factor (FGF) (Peprotech). All cells were cultured at $37^{\circ} \mathrm{C}$ with $5 \% \mathrm{CO}_{2}$ and negative for mycoplasma.

\section{Drug screen}

A drug screen was conducted using the Prestwick Chemical Library (Prestwick Chemical, Illkirch, France); a collection of 1120 drugs and small molecules, 95\% of which are approved for use in humans (FDA, EMA, and other agencies). U251 cells were seeded at a density of 75,000 cells $/ \mathrm{mL}$ and treated with $30 \mu \mathrm{M}$ of each drug for $1 \mathrm{~h}$ at $37^{\circ} \mathrm{C}$ and subjected to $2 \mathrm{~h}$ of hypoxia $\left(<1 \% \mathrm{O}_{2}\right)$ using a hypoxia incubator chamber (see below). Cells were fixed and stained for the SG marker TIAR $1 \mathrm{~h}$ postrelease from hypoxia to identify drugs interfering with SG dissolution. A second drug library was applied to U251 cells for the same time course but in normoxia. Plates were read using a Cellomics Arrayscan $\mathrm{V}^{\mathrm{TI}}$ automated fluorescence imager. Images were analyzed with Arrayscan software (Zeiss) and SGs counted by the ArrayScan $\mathrm{V}^{\mathrm{TI}}$ HCS Reader algorithm ${ }^{27}$; data was exported to and analyzed with Microsoft Excel. Drugs were considered positive hits if the mean number of granules per cell was two standard deviations (SD) from the mean of control cells. All 1120 drugs were ranked according to their $z$-scores (SGs present at $1 \mathrm{~h}$ normalized to non-drug controls). Final drug selection was based on a combination of deviation from control mean SGs per cell and literature linking them to either GBM treatment or a role in hypoxic or oxidative stress. None of the top candidate drugs were linked to SGs prior to our selection.

\section{Hypoxia}

U251 cells were seeded at a density of 100,000 cells $/ \mathrm{mL}$ and treated with a final concentration of $40 \mu \mathrm{M}$ raloxifene hydrochloride (Cayman Chemicals), $40 \mu \mathrm{M}$ chloroquine (CQ) (Sigma-Aldrich) or dimethyl sulfoxide (DMSO) vehicle control for $1 \mathrm{~h}$ at $37^{\circ} \mathrm{C}$. Hypoxia $\left(<1 \% \mathrm{O}_{2}\right)$ was induced using a hypoxia incubator chamber (STEMCELL Technologies). Immediately prior to placing cells in the hypoxia chamber, half of the media was removed from each well to limit the presence of dissolved oxygen. For control cells that did not undergo hypoxia, the same amount of media was removed but the cells were not placed in the hypoxia chamber and remained at $20 \% \mathrm{O}_{2}$ (normoxia). To induce hypoxia, the chamber was flushed with high purity (99.9\%) nitrogen gas at 2 psi for $10 \mathrm{~min}$, sealed and incubated at $37^{\circ} \mathrm{C}$ for $50 \mathrm{~min}$. This was followed by a second $10 \mathrm{~min}$ nitrogen gas flush and a $1 \mathrm{~h}$ incubation at $37^{\circ} \mathrm{C}$ (for a total of $2 \mathrm{~h}$ of hypoxia). U3024 MG primary cells were treated with raloxifene or DMSO vehicle control in serum-free DMEM (as Neurobasal media contains sodium pyruvate which prevents the formation of hypoxia-induced SGs; see Supplementary Fig. 4). U3024 cells received a total of $1 \mathrm{~h}$ hypoxia (10 min nitrogen gas flush plus $50 \mathrm{~min}$ incubation in the hypoxia chamber).

\section{Immunofluorescence}

Following hypoxia or the equivalent normoxia incubation, cells were fixed at various times in $4 \%$ paraformaldehyde for $10 \mathrm{~min}$. Following fixation, outer cellular membranes were stained with wheat germ agglutinin (WGA), alexa 647 conjugate (Thermo Fisher Scientific) diluted 1:400 in PBS for 10 min and permeabilized in 0.1\% Triton X-100 for $10 \mathrm{~min}$. Cells were blocked in 8\% BSA in PBS for $1 \mathrm{~h}$ followed by a $1 \mathrm{~h}$ incubation with antibodies specific for TIAR (BD Biosciences, 610352; diluted 1:200), G3BP2 (Sigma-Aldrich, HPA018304; diluted 1:1500), or FMRP (Cell Signaling Technology, 7104; diluted 1:400) in $1 \%$ BSA. Subsequently, cells were incubated with Alexa Fluor 488-chicken anti-mouse and Alexa Fluor 555donkey anti-rabbit (Thermo Fisher Scientific) secondary antibodies in 1\% BSA for $1 \mathrm{~h}$. Cell nuclei were counterstained with $1 \mu \mathrm{g} / \mathrm{ml} \quad$ 4',6-diamidino-2-phenylindole (DAPI, Sigma-Aldrich). Coverslips were mounted on frosted glass microscope slides using ProLong Gold antifade mounting media (Thermo Fisher Scientific) and allowed to cure overnight.

\section{Quantification of SGs}

Imaging: Imaging was performed on an AxioImager Z2 (Zeiss) microscope at $\times 40$ objective. Ten fields of view were obtained per timepoint \pm hypoxia, imaging $100+$ cells per condition. Exposure on all channels was unchanged through the imaging and intensity ranges of each image were identical. Images were exported as separate channel TIF files and quantified using CellProfiler.

CellProfiler: Image analysis was performed using CellProfiler (cellprofiler.org), an open source software for image analysis ${ }^{28}$. The pipeline used was designed as follows. Nuclei were detected as primary objects using automatic thresholding of the DAPI image. Cells were identified as secondary objects using the propagate function from the identified nuclei, determining the cell's outer edge in the WGA image. Following a series of enhancement and masking steps, puncta were measured in the cytoplasm of cells using a global thresholding with 
robust background adjustments. Puncta number per cell, intensities and locations were measured and exported as. csv files.

$R$ analysis: RStudio software was used to analyze CellProfiler output. Puncta counts per cell from two SG markers were determined and only puncta that had sufficient intensity measurements and $>50 \%$ correlation from both markers were considered SGs. Error and significance of puncta counts within a given experiment was determined using a negative binomial model, commonly used for count data with unequal variance.

\section{Western blot analysis}

Total cell lysates were harvested in Laemmli lysis buffer and protein concentration determined using the DC Protein Assay (Bio-Rad). Protein lysates were separated in 12\% TGX stain-free gels which were then activated for $45 \mathrm{~s}$ after SDS-electrophoresis, transferred to PVDF membranes using the Trans-Blot Turbo transfer system and imaged with the ChemiDoc Touch imaging system (Bio-Rad). Primary antibodies were used as follows: mouse anti-puromycin (1:8000; EMD Millipore, MABE343), rabbit anti-eIF2 $\alpha$ (1:1000; Cell Signaling Technology, 9722), rabbit anti-phospho-eIF2 $\alpha$ (Ser51) (1:500; Cell Signaling Technology, 9721), rabbit antiribosomal protein S6 (rpS6) (1:4000; Cell Signaling Technology, 2317), rabbit anti-phospho-rpS6 (Ser235/ 236) (1:4000; Cell Signaling Technology, 2211), rabbit anti-GADD34 (1:750; Thermo Fisher Scientific, PA1-139), rabbit anti-LC3B (1:1000; Cell Signaling Technology, 2775), mouse anti-SQSTM1/p62 (D5L7G) (1:1000; Cell Signaling Technology, 88588), mouse anti-G3BP1(1:250, Santa Cruz Biotechnology, sc-81940), rabbit anti-G3BP2 (1:2500; Sigma-Aldrich, HPA018304). Detection was performed with peroxidase-coupled secondary antibodies (Cell Signaling Technology) with Clarity Western ECL substrate (Bio-Rad). All blots were normalized to total lane protein and band intensities were quantified using ImageLab software (Bio-Rad).

\section{Annexin/PI flow cytometric analysis}

The Annexin V-Alexa Fluor 488/propidium iodide (PI) dead cell apoptosis kit (Thermo Fisher Scientific V13241) was used to detect early and late apoptosis and necrosis. Cells were treated with increasing doses of raloxifene $(40-100 \mu \mathrm{M})$ and $2 \mathrm{~h}$ following hypoxic or the equivalent normoxic incubation, cells were collected and stained with Annexin/PI according to manufacturer's protocol. Flow cytometry was performed using a BD FACSCanto II with 50,000 events being recorded per sample. Data was analyzed using Flowjo software. Based on forward and side scatter measurements cellular debris was gated out and all experimental data was compensated with single color controls for apoptosis (hydrogen peroxide) and necrosis (heat).

\section{CRISPR/Cas9 G3BP1 and G3BP2 knockouts}

CRISPR guide RNA (gRNA) sequences used in this study were selected and analyzed using the COSMID (CRISPR Off-target Sites with Mismatches, Insertions and Deletions) website (http://crispr.bme.gatech.edu/) to check for any potential off-target sites against the GRCh38 (hg38) genome build and are listed below:

\begin{tabular}{ll}
\hline CRISPR gRNA & Oligonucleotide sequence $\left(\mathbf{5}^{\prime}-\mathbf{3}^{\prime}\right)$ \\
\hline${ }^{29}$ G3BP1 gRNA oligo A & CACCGAAGCCTAGTCCCCTGCTGGT \\
G3BP1 gRNA oligo B & AAACACCAGCAGGGGACTAGGCTTC \\
G3BP2 gRNA oligo A & CACCGTGGCCATAAACAGCTTCCTG \\
G3BP2 gRNA oligo B & AAACCAGGAAGCTGTTATGGCCAC \\
non-targeting (nt) gRNA1 oligo A & CACCGGCACTACCAGAGCTAACTCA \\
non-targeting (nt) gRNA1 oligo B & AAACTGAGTAGCTCTGGTAGTGCC \\
non-targeting (nt) gRNA2 oligo A & CACCGCTCATCTATCGCGGTCGTC \\
non-targeting (nt) gRNA2 oligo B & AAACGACGACCGCGATAGATGAGC \\
& \\
\hline
\end{tabular}

CRISPR gRNA oligonucleotides were annealed and cloned into BbsI-digested lentiCRISPRv2 (Addgene plasmid \#52961). Lentivirus was produced by polyethylenimine (PEI)-mediated co-transfection of lentiCRISPR-gRNA with second generation lentiviral vectors pMD2.G and psPAX2 (Addgene plasmid \#12259 and \#12260, respectively) into human HEK-293T cells. Viral supernatant was collected $48 \mathrm{~h}$ post-transfection and used to transduce U251 cells in the presence of $5 \mu \mathrm{g} / \mathrm{ml}$ polybrene (Sigma-Aldrich) for $24 \mathrm{~h}$. To select for viral integration, cells were cultured in the presence of $1 \mu \mathrm{g} / \mathrm{ml}$ puromycin and individual clones were selected and screened for G3BP1 or G3BP2 knockout by western blot.

\section{Results}

\section{Correlation between mRNAs related to SG dynamics and} astrocytoma progression

TCGA contains RNA-Seq data from hundreds of human GBM samples; this database was interrogated with GlioVis for the mRNA expression levels of two hypoxiaresponsive kinases involved in the ISR (GCN2, PERK), as well as the SG aggregator G3BP1 and the inducible phosphatase of SG disassembly GADD34 in Grades II, III, and IV (GBM) astrocytomas. All four genes demonstrated increased expression from low-grade astrocytoma to GBM (Fig. 1A). Expression of these genes did not correlate with survival in GBM, however these genes did 


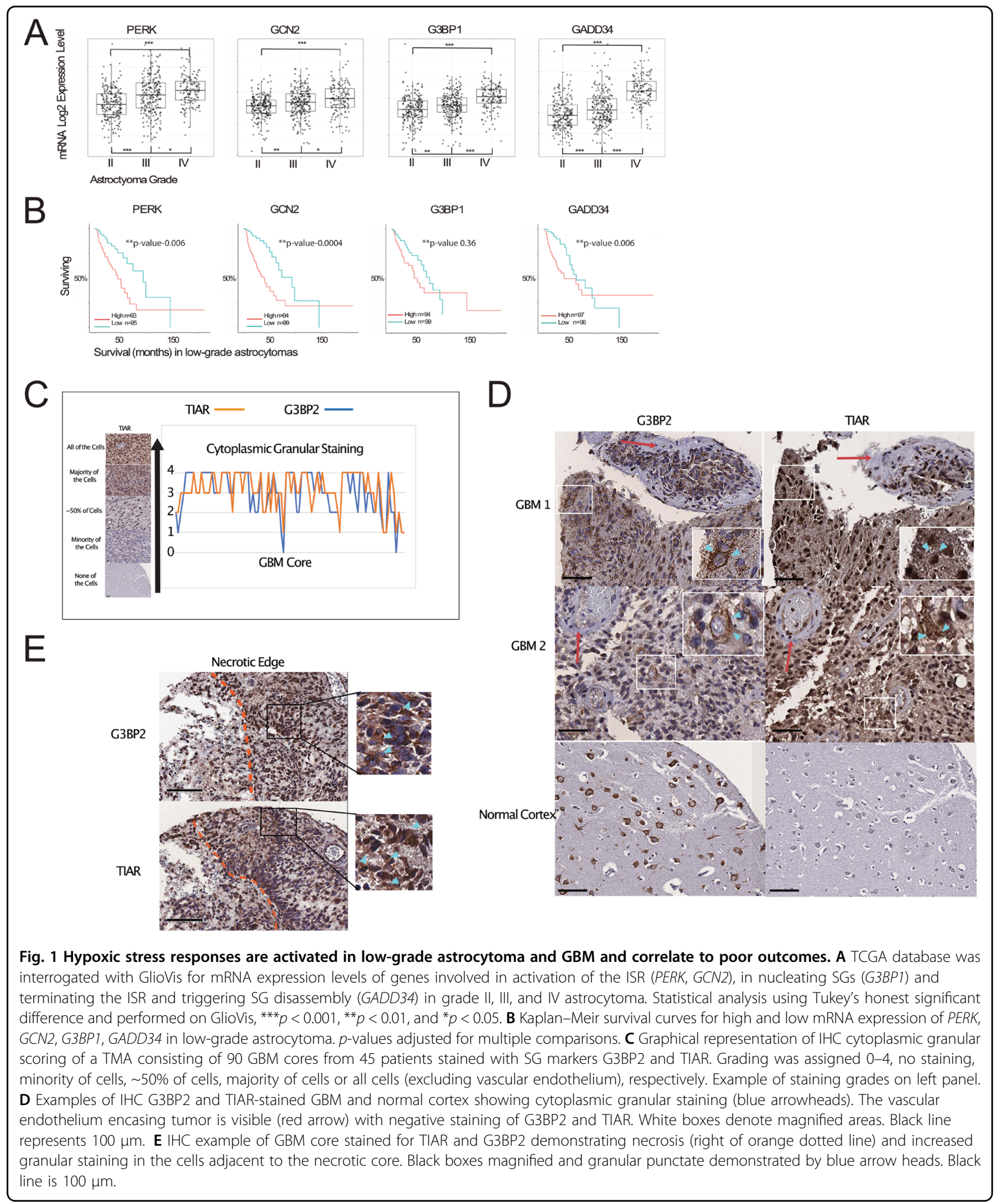

predict survival in low-grade astrocytoma with exception of G3BP1 which trended to significance (Fig. 1B). This suggests that the stress response is more active in GBM and in lower-grade astrocytomas with poorer prognosis. In support of an active SG response in GBM we immunohistochemically stained serial sections of a TMA with 
G3BP2 and TIAR. The majority of GBM samples demonstrated increased cytoplasmic punctate staining in at least some if not most of the core compared to the two normal controls (Fig. 1C, D). Although, the same cells could not be seen between TIAR and G3BP2 the same regions were visible and the correlation between G3BP2 and TIAR was high (Fig. 1C, D). In normal cortex, G3BP2-stained punctae in the cytoplasm of neurons but not the astrocytes and TIAR exhibited no cytoplasmic staining (Fig. 1D). Interestingly, necrotic regions were observed in 36 GBM cores and in $70 \%$ of these cores, GBM cells adjacent demonstrated high granular staining (Fig. 1E).

\section{A cellomics-based screen identified drugs that prevent SG dissolution post-hypoxia in GBM cells}

The detection of cytoplasmic puncta consistent with SGs in human GBM tissue combined with the observed correlation between astrocytoma progression and high expression of mRNAs related to SG dynamics lead us to further investigate the possibility of altering this pathway pharmacologically. A high-throughput cellomics-based screen of the Prestwick Chemical Library was conducted to identify drugs that impacted SG dissolution posthypoxic stress in U251 human GBM cells. The top 100 drugs with the highest $z$-score changes were identified, plotted, and colored by drug class (Fig. 2; Supplementary Table 1). The majority of the top 100 drugs (55\%) belonged to classes of drugs interfering with monoamine metabolism, however no obvious trends were apparent regarding specific drug classes. Nine drugs induced SGs in normoxia and were removed from further consideration (red circles Fig. 2). Guanabenz is a known drug which sustains eIF2 $\alpha$ phosphorylation and delays SG dissolution ${ }^{30}$; this drug served as a positive control in our screen and is denoted by the green dot in Fig. 2 . Candidate molecules with known links to GBM, hypoxia, oxidative stress, or SGs were retained for further analysis. Notably, the SERMs raloxifene and clomiphene, which are auxiliary therapeutic modalities in $\mathrm{GBM}^{31,32}$, both delayed SG dissolution in U251 cells post-hypoxia (black circles Fig. 2).

\section{Raloxifene delays dissolution of hypoxia-induced SGs in GBM cells}

As secondary validation, we conducted a dose response curve of raloxifene's effect on hypoxia-induced SGs by treating U251 cells with escalating doses of raloxifene $(0-60 \mu \mathrm{M})$. Doses as low as $20 \mu \mathrm{M}$ prevented SG dissolution post-hypoxia, while maximal suppression of SG dissolution was observed at 50-60 $\mu \mathrm{M}$ (Fig. 3A). Conversely, raloxifene did not induce SGs at any dose in U251 cells cultured in normoxic conditions (Fig. 3A). A dose of $40 \mu \mathrm{M}$ was selected for subsequent testing as it was the median dose that elicited a significant SG response.

Sustained SGs in the presence of raloxifene was visually confirmed by dual TIAR and G3BP2 immunofluorescence staining in both the immortalized GBM cell line U251, in addition to a primary GBM cell line U3024 (top panels Fig. 3B, C). Raloxifene alone did not induce SGs in either cell line during normoxia (bottom panels Fig. 3B, C).

To describe our findings in a more objective manner, we developed an automated image analysis pipeline in CellProfiler (see "Materials and methods" section) to quantify the percentage of cells containing SGs, SG number per cell, and SG intensity (used as a correlative measurement for SG size). Immediately post-hypoxia, U251 cells exhibited a robust SG response irrespective of the presence of raloxifene, with no significant difference in percentage of cells exhibiting SGs, SGs/cell, or SG intensity (Fig. 4A-C). Complete SG dissolution occurred within 15-30 min post-hypoxia in control cells; however, the rate of SG dissolution following hypoxia was significantly slower in cells pre-treated with raloxifene (Fig. 4A, B). These results were further confirmed in the U3024 primary GBM cell line. Similar to U251s, raloxifene pretreatment in these cells leads to sustained SGs (Fig. 4D, E); however, the initial percentage of cells with SGs is significantly higher with raloxifene pre-treatment $(92 \%$ and $65 \%$, respectively, Fig. 4D).

\section{Raloxifene inhibits resumption of bulk translation post- hypoxia}

SG formation is traditionally coupled with stressinduced translational silencing, therefore we wanted to determine if raloxifene-induced granulostasis exhibited differences in global protein translation compared to a normal SG response. To measure global protein synthesis levels, raloxifene and vehicle-treated cells were pulsed with puromycin at various times post-hypoxia. Puromycin incorporation into nascent polypeptide chains was then visualized by western blot, quantified, and used as an indicator of overall protein synthesis. A steady increase in protein translation can be observed in control cells posthypoxia, concomitant with loss of SGs (Figs. 5A and 4). However, immediately post-hypoxia, protein translation was significantly decreased in cells pre-treated with raloxifene (Fig. 5A) and remained low for the duration of the $2 \mathrm{~h}$ stress recovery period, correlating with the persistence of SGs in these cells (Fig. 4). Importantly this was not due to raloxifene itself impacting protein translation, as raloxifene-treated cells that were maintained in normoxia for the same duration of time displayed no difference in puromycin incorporation relative to vehicle control cells (Fig. 5B). See Supplementary Fig. 1 for whole western blot images. 


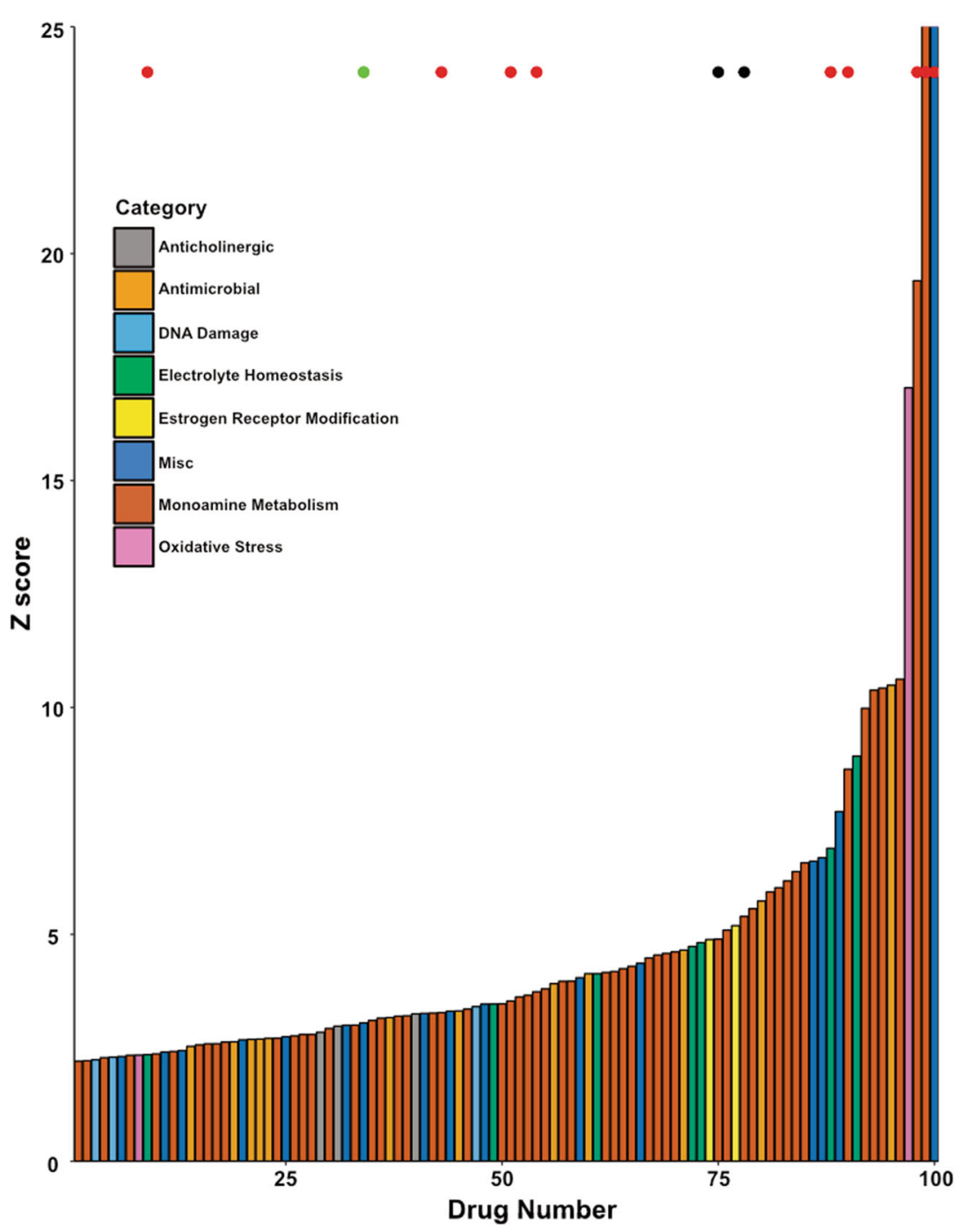

Fig. 2 A high-throughput screen identifies drugs showing significant inhibition of SG dissolution $\mathbf{1}$ h post-hypoxia. U251 human GBM cells were treated with drugs from the Prestwick Chemical Library (1120 drugs, $30 \mu \mathrm{M}$ each) or vehicle controls for $1 \mathrm{~h}$ and exposed to $2 \mathrm{~h}$ of hypoxia $\left(\sim<1 \% \mathrm{O}_{2}\right)$. Cells were returned to normoxia for $1 \mathrm{~h}$ and subsequently fixed and immunostained for TIAR. As a control U251 cells were treated with the library in the absence of hypoxia. SGs were counted and all 1120 drugs were ranked according to their z-scores. The top 100 drugs are displayed in order of ascending effect as determined by $z$ score (SGs present at $1 \mathrm{~h}$ normalized to non-drug controls), and bars are color-coded by drug class. Red dots denote drugs having a high likelihood of being false positives. The green dot represents guanabenz, a known inhibitor of SG dissolution (positive control). Black dots represent the SERMs raloxifene and clomiphene.

Canonical SG presence requires phosphorylation of the $\alpha$ subunit of eIF $2 \alpha$, an event which blocks translation initiation $^{33,34}$. In correlation with the observed decrease in global protein translation, raloxifene treatment resulted in sustained eIF $2 \alpha$ phosphorylation post-hypoxia. This was in direct contrast to control cells in which eIF $2 \alpha$ phosphorylation diminished with increasing global translation and loss of SGs (Fig. 5C). Raloxifene alone was not responsible for this phosphorylation event as raloxifene and vehicle-treated cells that were not made hypoxic showed no difference in phospho-eIF2 $\alpha$ (Fig. 5D).
Dephosphorylation of phospho-eIF2 $\alpha$ is accomplished by stress-induced cellular upregulation of the phosphatase GADD34. We initially suspected that failed upregulation of GADD34 could explain the sustained phospho-eIF2 $\alpha$ observed in raloxifene-treated cells. Surprisingly, GADD34 protein levels do not change between raloxifene and control cells post-hypoxia (Fig. $5 \mathrm{C}$ ) suggesting granulostasis, and that sustained eIF $2 \alpha$ phosphorylation and concomitant SGs are occurring through a different mechanism. See Supplementary Figs. 1 and 2 for whole western blot images. 
A

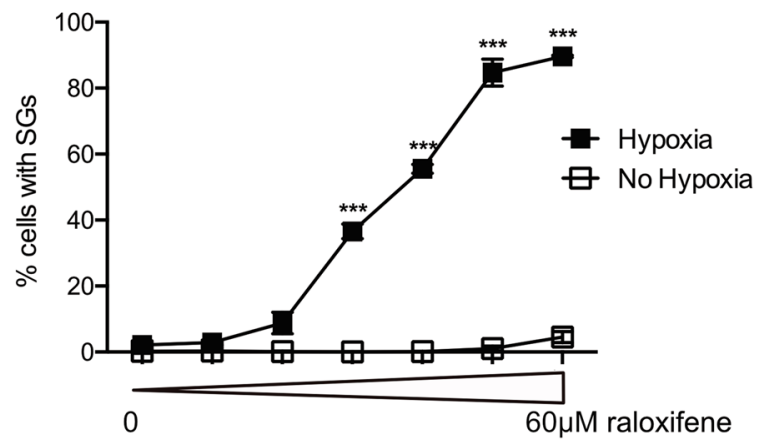

B

DMSO

Raloxifene
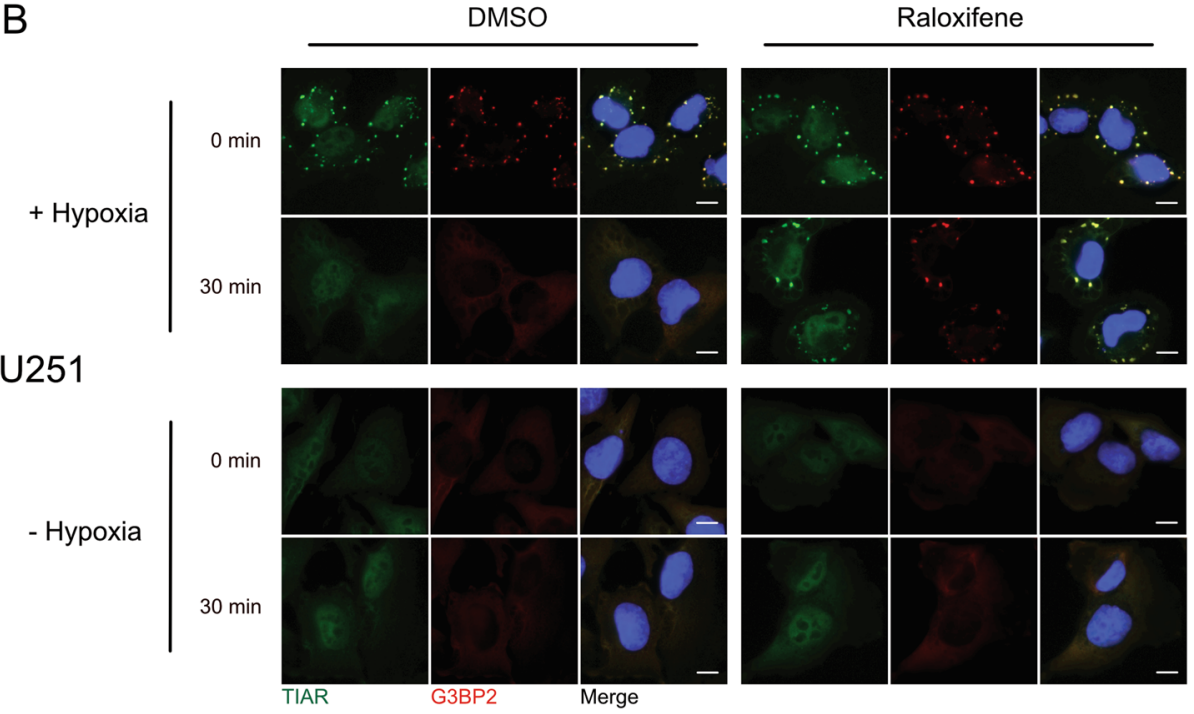

C

DMSO

Raloxifene
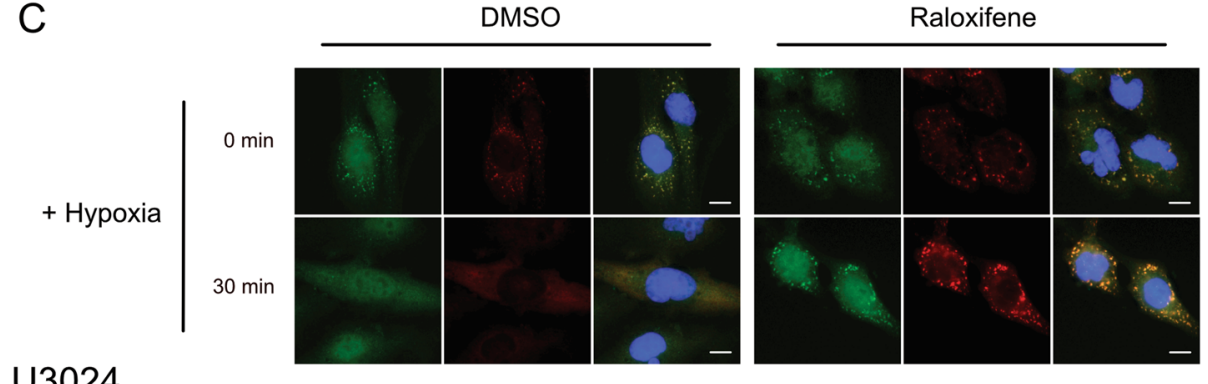

U3024
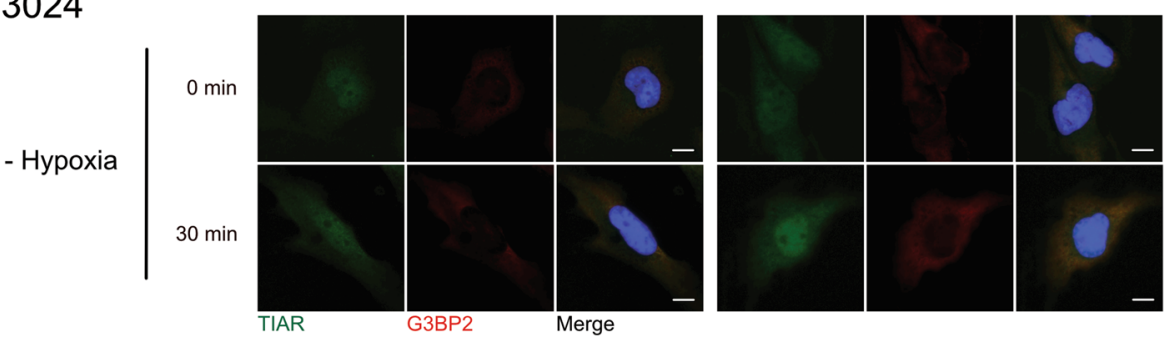

Fig. 3 Raloxifene inhibits dissolution of hypoxia-induced SGs in glioblastoma cells and does so in a dose-dependent manner. A Raloxifene dose response curve. U251 glioblastoma cells were treated with raloxifene at doses ranging from 0 to $60 \mu \mathrm{M}$ for $1 \mathrm{~h}$ prior to a $2 \mathrm{~h}$ incubation \pm hypoxia $\left(<1 \% \mathrm{O}_{2}\right)$. Cells were allowed to recover for $1 \mathrm{~h}$ in normoxia and then fixed and stained for SG markers TIAR and FMRP. Percentage of cells with SGs were counted manually; 10 fields of view $\sim 50$ cells per field. Data is presented as the mean of triplicates \pm SEM, unpaired $t$-test ${ }^{* * *} p<0.001$. B Immortalized GBM U251 cells and C primary GBM U3024 cells were treated with $40 \mu \mathrm{M}$ raloxifene or vehicle control (DMSO) for $1 \mathrm{~h}$ followed by a $2 \mathrm{~h}$ (U251) or $1 \mathrm{~h}$ (U3024) incubation \pm hypoxia $\left(<1 \% \mathrm{O}_{2}\right)$. Cells were then fixed immediately ( $0 \mathrm{~min}$ ) or allowed to recover (30 min) in normoxia before being fixed and stained for SG markers TIAR (green) and G3BP2 (red). DNA was counterstained with DAPI (blue). Scale bars $=10$ microns. 


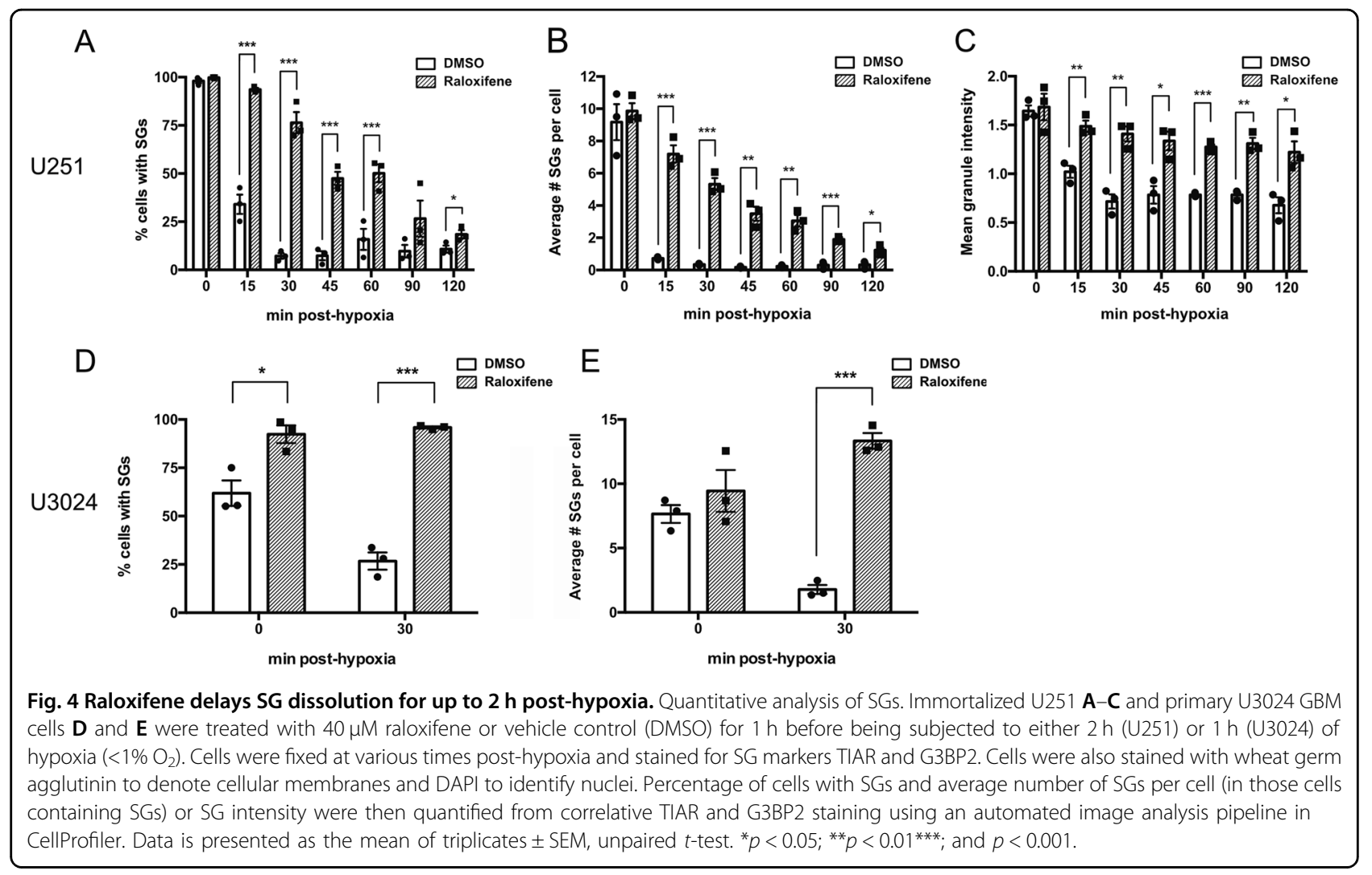

\section{Raloxifene prevents re-activation of $\mathrm{mTOR}$ signaling post- hypoxia}

Mammalian target of rapamycin (mTOR), is a key GBM oncogene that phosphorylates a wide variety of substrates involved in stress recovery and cancer pathogenesis. During stress, mTOR function is diminished to conserve cellular energy ${ }^{35,36}$. We previously demonstrated that mTOR effectors are contained in $\mathrm{SGs}^{37}$ and therefore postulated that mTOR activity would be low in U251 cells with sustained SGs. To ascertain the status of mTOR signaling in our model, we examined the phosphorylation level of the mTOR effector rpS6 in raloxifene-treated cells post-hypoxia. rpS6 phosphorylation was decreased up to $2 \mathrm{~h}$ post-hypoxia in raloxifene-treated cells relative to control cells (Fig. 5C) in correlation with sustained SGs. No change was detected in phospho-rpS6 levels between raloxifene and control cells that were maintained in normoxia (Fig. 5D). See Supplementary Fig. 2 for whole western blot images.

The combination of raloxifene and hypoxia results in GBM cell death and in autophagic inhibition similar to $\mathrm{CQ}$

The addition of raloxifene prior to hypoxic stress appears to prolong stress recovery even after cells are returned to normoxia. Without the ability to resolve stress, we postulated that cell viability would be adversely impacted. Cell death was evaluated by determining levels of early and late apoptosis/necrosis with AnnexinV/PI staining. Increasing doses of raloxifene in combination with hypoxia displayed a significant shift in the number of cells undergoing late apoptosis/necrosis (Fig. 6A, B). Importantly while the amount of apoptosis occurring in raloxifene-treated cells was higher than vehicle control or hypoxia alone, it did not exhibit the shift towards late apoptosis/necrosis seen with the combination of raloxifene and hypoxia (Fig. 6A).

It is known that autophagy is required for clearance of retained SGs and there is cross-talk between apoptosis, necrosis, and autophagy ${ }^{38}$, therefore we asked whether defects in autophagy could account for retained SGs. LC3II is a protein required during the expansion/elongation phase of autophagy. LC3-II levels also increase when the fusion of autophagosomes with lysosomes is inhibited, such as by treatment with the known lysosomal inhibitor $\mathrm{CQ}^{39}$. Raloxifene treatment with or without hypoxia lead to significantly increased LC3-II levels (Fig. 7A, B), comparable to CQ (Fig. 7C, D). Similarly, increases in p62 are associated with a block in autophagy and were also observed with raloxifene and CQ treatment (Fig. 7A-D). Interestingly the combination of hypoxia and CQ also resulted in sustained SGs (Fig. 7E). See Supplementary Fig. 5 for whole western blot images.

As raloxifene is a SERM, we initially attempted to determine whether raloxifene-induced granulostasis and 

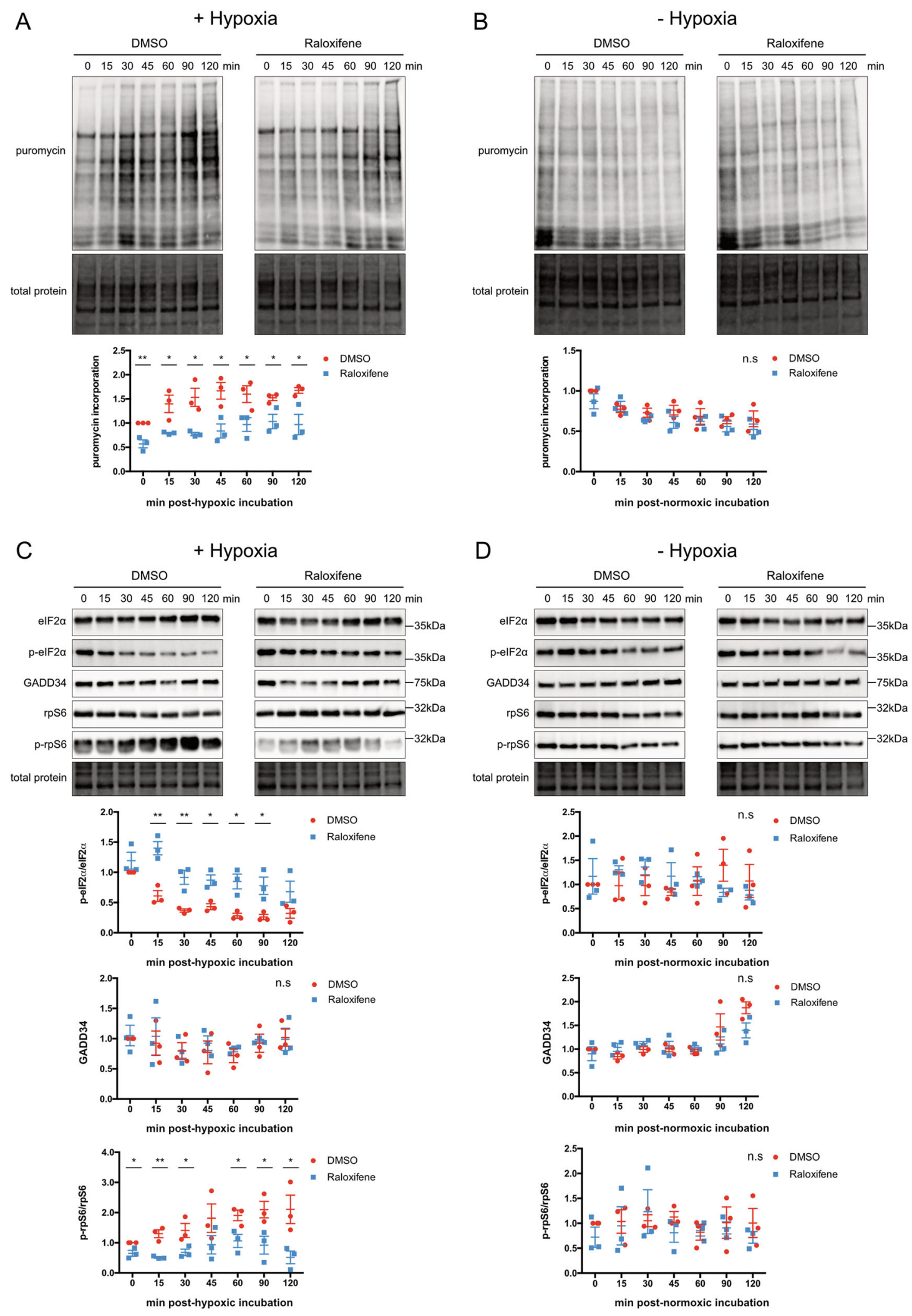

Fig. 5 (See legend on next page.) 
(see figure on previous page)

Fig. 5 Raloxifene delays resumption of protein translation post-hypoxia in correlation with increased elF2a phosphorylation and decreased mTOR signaling. A and B Puromycin incorporation assay. U251 cells were treated with $40 \mu \mathrm{M}$ raloxifene or DMSO vehicle control for $1 \mathrm{~h}$ prior to $2 \mathrm{~h}$ hypoxic $\mathbf{A}$ or non-hypoxic $\mathbf{B}$ incubation. At various times post-hypoxic or normoxic incubation cells were treated with $10 \mu \mathrm{g} / \mathrm{mL}$ puromycin for $10 \mathrm{~min}$ and puromycin incorporation into nascent proteins was detected by anti-puromycin blot. Blots were normalized to total lane protein, and represented as ratios with DMSO time 0 normalized to 1 (bottom quantification panels). Data is presented as the mean of triplicates \pm SEM, unpaired t-test ${ }^{*} p<0.05 ;{ }^{* *} p<0.01$. C and $\mathbf{D} \cup 251$ cells were treated as in $\mathbf{A}$ but without addition of puromycin. Cell lysates were probed for elF2a, phospho-elF2a, GADD34, rpS6, and phospho-rpS6 at various times post-hypoxic $\mathbf{C}$ or normoxic $\mathbf{D}$ incubation. All blots were normalized to total lane protein with DMSO time 0 set to 1 (bottom quantification panels). The level of elF2a phosphorylation is presented as the ratio of $p$-elF2a to total elF2a (bottom quantification panels). The level of rpS6 phosphorylation is similarly presented. Data is presented as the mean of triplicates \pm SEM, unpaired $t$-test, and false discovery rate of $1 \% .{ }^{*} p<0.05 ;{ }^{* *} p<0.01$.

cell death was via antagonistic or agonistic effects at the estrogen receptor. No concentration of $\beta$-estradiol could mimic or rescue granulostatic effect (Supplementary Fig. 3). We also attempted to rescue SG stasis with the addition of the anti-oxidant pyruvate, however this failed to prevent granulostais suggesting raloxifene is not acting as a pro-oxidant (Supplementary Fig. 4$)^{40,41}$.

\section{G3BP1 knock-out partially restores SG dissolution in raloxifene-treated cells}

A recent publication suggested G3BP1 may be required for SG dissolution during hyperactive stress ${ }^{42}$. G3BP1 and G3BP2 are SG aggregating proteins that are critically important for SG assembly and loss of both are required to inhibit SG formation in response to several stressors, but not hypoxia ${ }^{29,43}$. We generated U251 G3BP1 and G3BP2 null cell lines using a CRISPR lentiviral delivery system (Fig. 8A). We pre-treated G3BP1 null, G3BP2 null, wild-type U251, and a non-targeting gRNA control (NTC) cell line with raloxifene or vehicle control prior to hypoxia. Interestingly, while G3BP2 null cells behaved largely like wild-type and NTC cells, the loss of G3BP1 partially restored SG dissolution in raloxifene-treated cells post-hypoxia (Fig. 8B, C).

\section{Discussion}

GBM is a highly aggressive tumor with a high rate of growth and invasiveness into the normal brain. These malignant tumors exist in a hostile microenvironment due to cells outstripping their local nutrient supplies (oxygen, glucose, amino acids) and the addition of chemotherapeutic-induced and radiation-induced stress. Despite these stressors, GBM cells continue to thrive and overcome treatment, resulting in a life expectancy of 14-16 months post-diagnosis ${ }^{1}$. This implies GBM cells have the ability to adapt to microenvironmental stressors. Emerging evidence suggests that stress adaptation happens through proteomic reprogramming at the translational level ${ }^{18,19}$. One mechanism of stress reprogramming is the sequestration of mRNAs and proteins into SGs.
Mining hundreds of RNA-Seq samples from human GBMs in TCGA databases demonstrates that mRNAs involved in the ISR and both SG formation and dissolution are upregulated in GBM compared to lower-grade, less aggressive astrocytomas (Fig. 1). Although mRNA expression did not predict survival in human patients with GBM, these mRNAs did predict poor survival when upregulated in the less aggressive, lower grade astrocytomas. It is known that all lower grade astrocytomas progress to their more malignant counterparts over time, in fact patients do not die from their low-grade astrocytoma but from malignant transformation. We hypothesize that lower-grade astrocytomas that demonstrate upregulation of these stress response genes may be exhibiting micro-areas of increased metabolic stress (nutrient starvation, hypoxia, etc.) that mark the beginning of malignant transformation, hence high expression relates to poor survival. Perhaps, upregulation of these stress-response genes can be used as markers of progression. Although provocative, this would need to be confirmed in a prospective manner with pathological correlates and protein data.

Our previous work demonstrated that SGs could form in GBM stem cells and culture in vitro ${ }^{37}$. Here we have demonstrated for the first time the presence of cytoplasmic aggregates reminiscent of SGs in operative human GBM tissues. Although normal cortical neurons displayed punctae of G3BP2 the background astrocytic cells did not, G3BP is known to form punctae in neuronal cell bodies ${ }^{44}$. Intriguingly cells near areas of necrosis demonstrated more intracellular TIAR and G3BP2 aggregates by visual inspection (Fig. 1). These areas of necrosis and the "pseudo-pallisading" adjacent cells are known to be hypoxic ${ }^{45}$. Although a promising indication that SGs exist in GBM and may be important in adaptation to environmental stressors, current technical limitations prevents us from fully characterizing these granules and confirming their identity as SGs (that they also contain RNA).

Given that TCGA data demonstrates that both the kinases that result in SG aggregation and the phosphatase that triggers SG dissolution are upregulated in GBM, we 


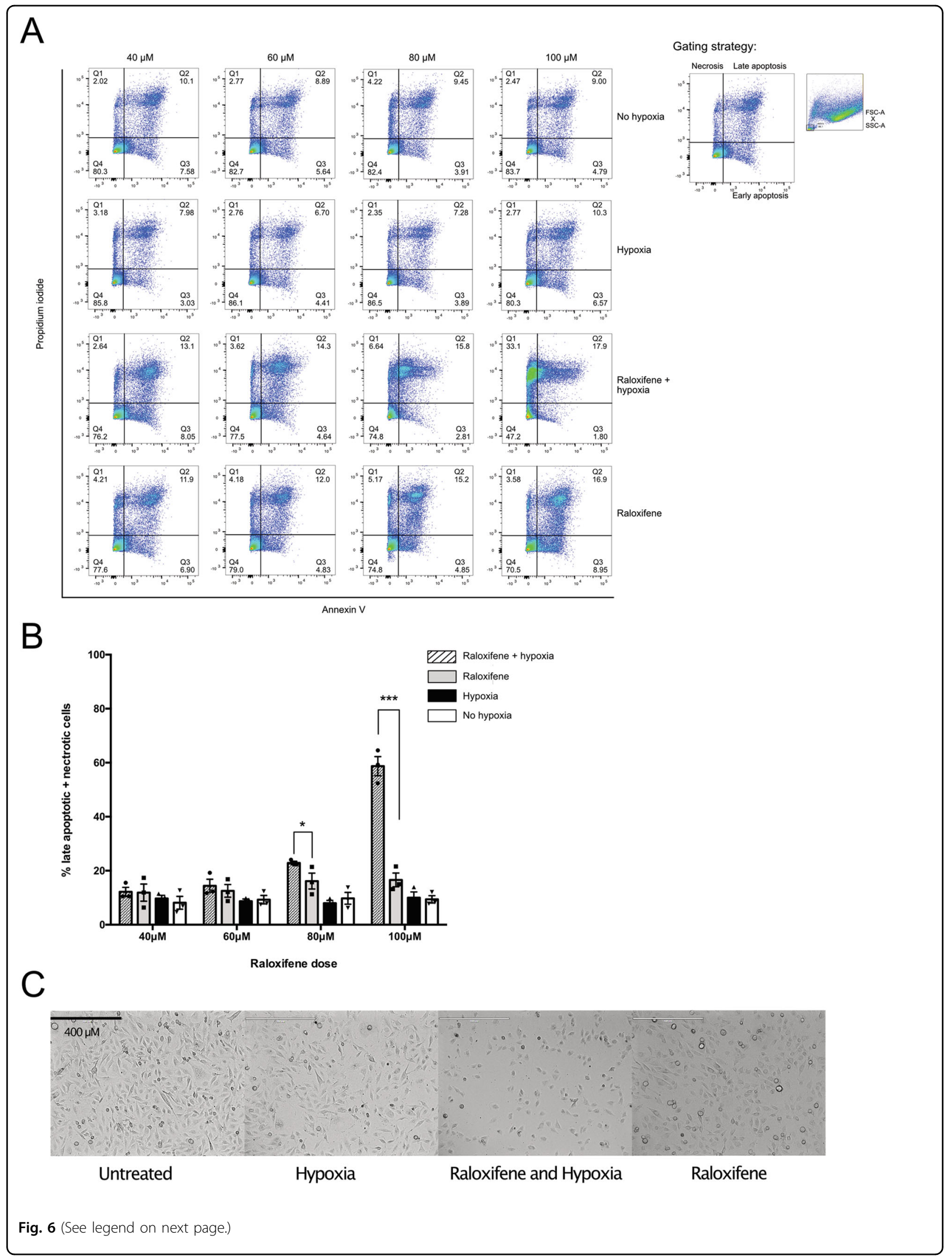


(see figure on previous page)

Fig. 6 Raloxifene results in increased cell death with hypoxia. A U251 cells were treated with raloxifene (or equivalent vehicle control) at doses ranging from 40 to $100 \mu \mathrm{M}$ for $1 \mathrm{~h}$ prior to a $2 \mathrm{~h}$ incubation \pm hypoxia $\left(<1 \% \mathrm{O}_{2}\right)$. Cells were allowed to recover for $2 \mathrm{~h}$ in normoxia, labeled live with annexin $\mathrm{V}$ and $\mathrm{Pl}$ and analyzed by flow cytometry. Cellular debris based on forward and side scatter was excluded from analysis (see right inset). Cells in the lower left (Q4) quadrant (annexin and PI negative) were classified as living, the lower right (Q3) quadrant (annexin positive) as early apoptotic, the upper right (Q2) quadrant (annexin and PI positive) as late apoptotic and cells in the upper left (Q1) quadrant (PI positive) were considered necrotic (see right inset). Representative dot plots display 50,000 events of annexin $\vee$ versus PI for each concentration and condition. B Percentage of late apoptotic and necrotic populations were combined and presented graphically as the mean of triplicates \pm SEM, unpaired $t$-test and false discovery rate of $1 \%$. ${ }^{*} p<0.05$; ${ }^{* *} p<0.01 ;{ }^{* *} p<0.001$. C Brightfield microscopy of vehicle control and raloxifene-treated $(40 \mu \mathrm{M})$ cells $2 \mathrm{~h}$ post \pm hypoxia.

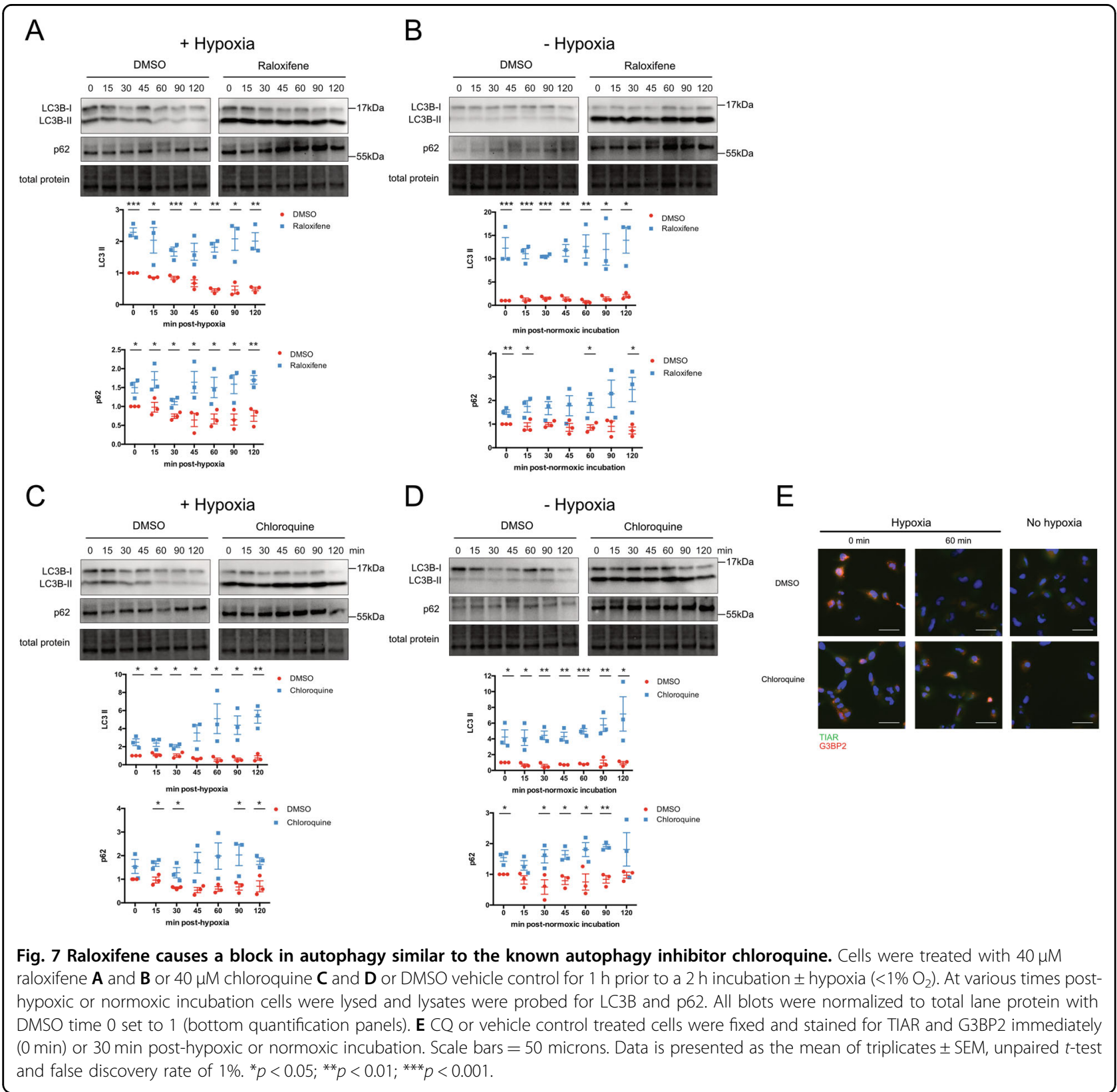

postulated that the cycling of SGs may be important in GBM cellular adaptation. Therefore, we hypothesized that pharmacologically impairing SG dissolution and restoration of translation would affect cellular homeostasis. This is consistent with observations in other disease conditions, such as viral infection and neurodegenerative disease where 

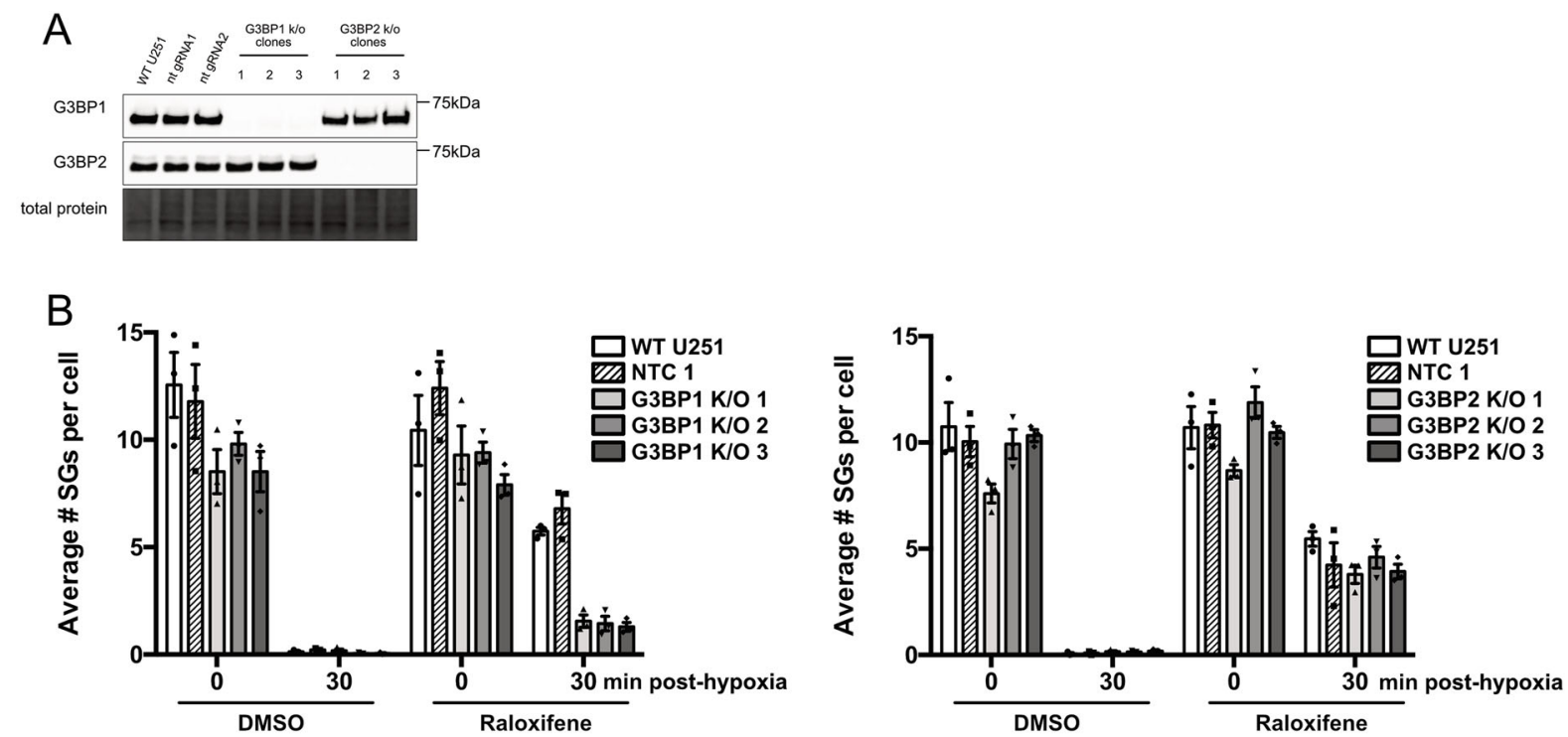

C

DMSO

Raloxifene
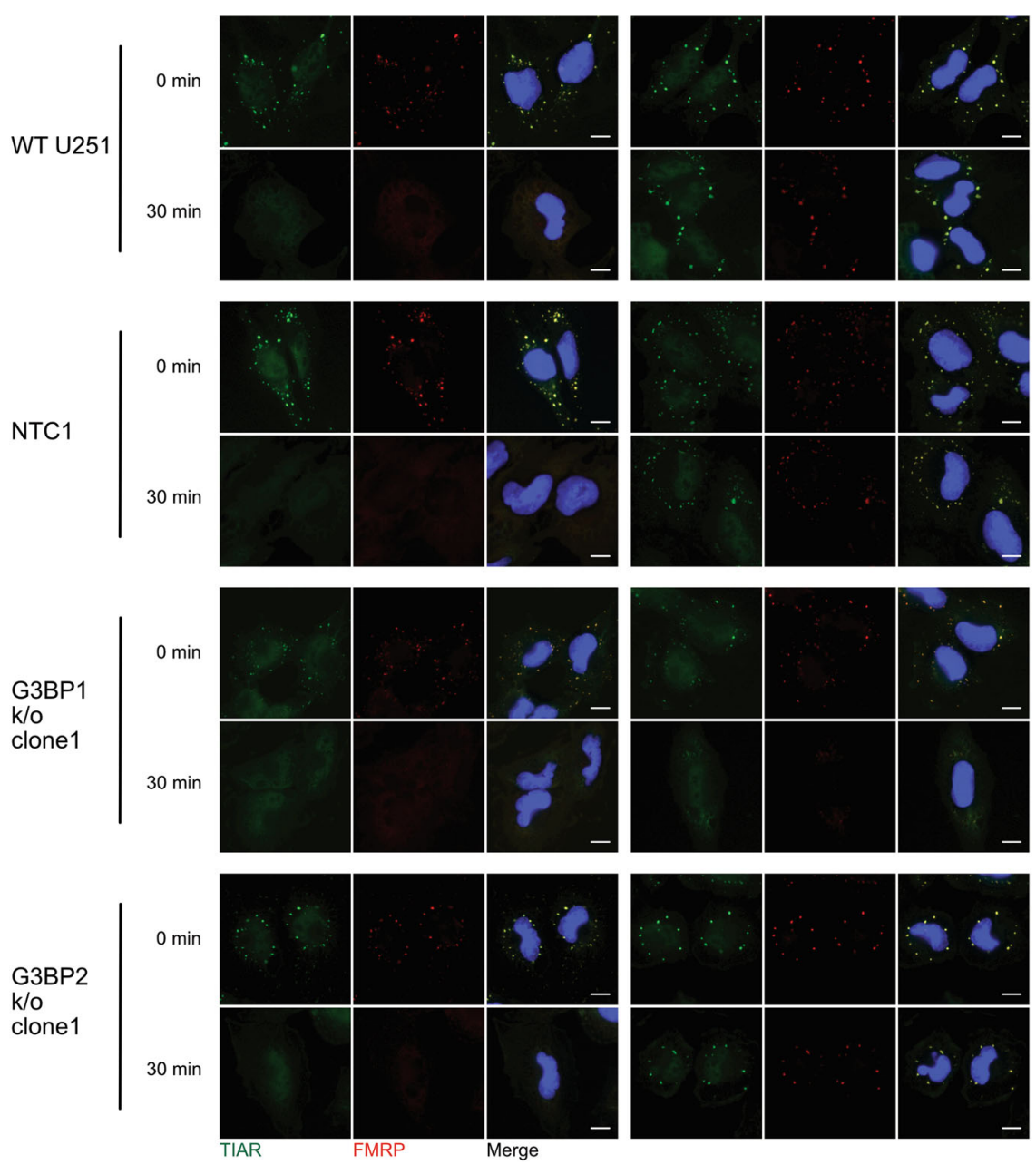

Fig. 8 (See legend on next page.) 
(see figure on previous page)

Fig. 8 G3BP1 knockout partially reverses raloxifene-induced delay of SG dissolution. A U251 cell lines stably expressing CRISPR gRNAs targeting G3BP1 or G3BP2 along with non-targeting (nt) gRNAs were established using a lentiviral-based system. B Untransduced (WT) U251 and nt gRNA control (NTC) cells along with G3BP1 and G3BP2 knockout clones were treated with $40 \mu \mathrm{M}$ raloxifene or DMSO vehicle control prior to $2 \mathrm{~h}$ of hypoxia $\left(<1 \% \mathrm{O}_{2}\right)$. Cells were fixed either immediately $(0 \mathrm{~min})$ or $30 \mathrm{~min}$ post-hypoxia. Cells were stained for CellProfiler analysis as previously described, and average number of SGs per cell (in those cells containing SGs) were quantified from correlative TIAR and FMRP staining in CellProfiler. Data is presented as the mean of triplicates \pm SEM. C Representative immunofluorescence images. Cells were stained for SG markers TIAR (green) and FMRP (red), and DNA was counterstained with DAPI (blue) immediately (0 min) or $30 \mathrm{~min}$ post-hypoxia. Scale bars $=10$ microns.

the dissolution and cycling of SGs are important ${ }^{46-49}$. To explore our hypothesis, we screened chemical compounds that resulted in granulostasis in U251 GBM cells after recovery from hypoxia-induced stress. Hypoxia was chosen as a stressor as GBM experiences high degrees of hypoxia which has been shown to promote tumor aggressiveness, stemness, invasion, and resistance to therapy ${ }^{50-52}$. Our screen identified 100 compounds with granulostasis and $z$ scores of $>2.2$ when compared to our negative controls (Fig. 2; Supplementary Table 1). We secondarily validated the drug raloxifene as having a dose-dependant ability to cause granulostasis in both an immortalized and a primary GBM stem cell line (Figs. 3 and 4). This was interesting to us as SERMs have been used as salvage chemotherapy with some effect in GBM and have been postulated to be radiation and chemotherapy sensitizers ${ }^{53,54}$

Typically, SG formation results in high levels of phospho-eIF2 $\alpha$, translational repression ${ }^{55}$, and suppression of mTOR activation ${ }^{55-58}$. We have demonstrated that following hypoxia, control cells dissolve SGs, dephosphorylate eIF $2 \alpha$, and restore translation and mTOR activity within $15 \mathrm{~min}$. Raloxifene treatment delays these processes at a rate coincident with SG dissolution beginning at around $1.5-2 \mathrm{~h}$ following release from hypoxia (Fig. 5A, C). mTOR is a key oncogene that drives GBM pathogenesis and is constitutively active in U251 cells due to an upstream frameshift mutation in the tumor suppressor PTEN ${ }^{59}$. It is significant that we were able to alter restoration of homeostasis and mTOR activity after hypoxia by delaying SG dissolution, albeit for a short-interval of time. We believe this lack of mTOR activity is due to translational silencing during granulostasis, however a direct inhibition of mTOR by raloxifene has not been ruled out and these experiments could be repeated in the presence of an mTOR inhibitor such as torin. However, if we could harness this dampening effect of mTOR for longer, this alone would be of significant clinical value as mTOR is a major GBM oncogene. This could be accomplished by either altering raloxifene for a more prolonged effect or by changing delivery methods (sustained release, decreasing dosing intervals).

Since the stress-induced phosphatase GADD34 dephosphorylates phospho-eIF $2 \alpha$, we questioned whether granulostasis resulted from failed GADD34 induction. We found no changes in GADD34 levels between hypoxia alone or raloxifene/hypoxia (Fig. 5C). Whether raloxifene impairs GADD34's ability to associate with phosphoeIF2 $\alpha$ remains to be tested but is a possibility.

Cycling of SGs is important to cell survival. If SGs are indeed a targetable pathway for GBM treatment, then it must follow that raloxifene is able to increase astrocytoma cell death. Consistent with this, increasing doses of raloxifene when combined with hypoxia lead to increasing granulostasis and a shift towards late apoptosis and necrosis (Fig. 6). We demonstrated that raloxifene treatment caused a block in late autophagy, as indicated by the accumulation of LC3-II over time of treatment (Fig. 7A, B). The LC3-II accumulation we observed was nearly identical to cells treated with the known autophagy blocker, CQ (Fig. 7C, D). We also observed that subjecting untreated cells to hypoxic conditions increased LC3-II levels, consistent with previous reports, and that these levels returned to baseline upon return to normoxia. ${ }^{60,61}$ However, treatment with raloxifene posthypoxia caused LC3-II and p62 steady-state levels to remain high, even upon restoration of normoxia. These observations are consistent with the following model: hypoxia increases autophagic flux but a late stage blockage in flux induced by CQ or raloxifene causes a failure of lysosomal degradation, and a shift towards necrosis. Another interesting observation was that raloxifene treatment alone did not result in granulostasis, alter translation or induce phospho-eIF $2 \alpha$ and phosphorpS6 levels compared to vehicle controls, suggesting two important interpretations. The first is that the stressful event of hypoxia exposure is required to initiate a sequence of events (eIF2 $\alpha$ phosphorylation, SG formation, and enhanced autophagic flux) aimed at promoting cell survival. When raloxifene is added to this 'stressed' system, the cell fails to survive. The second is that because hypoxia and raloxifene work synergistically to induce cell death, raloxifene treatment is less likely to be toxic to normoxic/healthy tissues. Provocatively, SERMs prevent oxidative stress in the brains of ovariectomized rats $^{62}$ and have been implicated as a neuro-protective agent in aging of the brain ${ }^{63}$. This potential dichotomy of raloxifene will need to be further explored utilizing humanized mouse models of GBM. 
Because CQ is a known inhibitor of autophagic lysosomal degradation ${ }^{39}$, we wanted to determine if $C Q$ worked similarly to raloxifene. CQ exhibited a similar phenomenon; hypoxia-induced SGs were retained after CQ as observed in raloxifene-treated cells (Fig. 7E). Previous work has shown that $\mathrm{CQ}$ can trigger cell death independent of apoptosis in GBM due to accumulation of autophagasomes ${ }^{39,64,65}$. Typically, SGs are cleared by the PQC and the HSP8-BAG3-HSP70 complex ${ }^{14}$ but as granulostasis persists SGs are cleared by autophagy ${ }^{16,38}$. In keeping with this, a block in late autophagy can result in SG accumulation as we observed in both raloxifene/ hypoxia and CQ/hypoxia-treated and cells (Figs. 4, 7D). Taken together, these data suggest that raloxifene is blocking late autophagy at a stage similar to $C Q$, resulting in unresolved autophagosome accumulation and eventual shift to necrosis. In future studies, we will further interrogate this mechanisms by staining for autophagosomes, knocking down canonical autophagy regulators (ATG5) and using chemicals that inhibit different stages of autophagic flux (torin, wortmannin, 3-MA).

We had previously generated G3BP1 and G3BP2 knockout U251 cell lines. These cells still form SGs in the presence of hypoxia as both G3BP1/2 need to be lost to prevent hypoxia-induced SG formation, similar to what has been described previously ${ }^{29}$. Interestingly, loss of G3BP1 but not G3BP2 resulted in partial rescue of raloxifene/ hypoxia-induced granulostasis (Fig. 8). This is intriguing as acetylation of G3BP1 at lysine-376 regulated by histone deacetylase-6 (HDAC6) has been directly linked to SG dissolution during hyperactive stress ${ }^{42}$. One possibility is that raloxifene alters acetylation of G3BP1 resulting in failure of SG dissolution and that loss of G3BP1 (perhaps G3BP1 acts a dominant negative) allows G3BP2 to take more of an active role in dissolution. Interestingly HDAC6 and autophagy are linked ${ }^{66,67}$, further supporting that G3BP1 and HDAC6 have a role in the SG stasis induced by raloxifene/hypoxia. These experiments are on-going in our laboratory.

SERMs have been shown to be effective against GBM in vitro as a chemo- and radiation sensitizer, and to have select activity in a subset of GBM patients. Although clinical trials of SERMs alone have not been effective, one wonders whether SERMs such as raloxifene may be more effective if combined with a chemotherapeutic that induces SGs (such as bortezomib ${ }^{68}$, vinca alkaloids, or 5fluorouracil) or other late blockers of autophagy. In addition, it is important to recognize that raloxifene may not be the best compound to exert SG stasis, but it works as a proof-of-concept that altering SG dynamics impairs cellular homeostasis after stress. Our screen results (100 potential candidates) demonstrates that SG cycling is a druggable pathway and other candidates should be investigated alone or in combination for a more robust effect on GBM cell viability. We have provided evidence that this pathway deserves further exploration as a viable therapeutic target.

\section{Acknowledgements}

This work was supported by grants from Dalhousie Department of Surgery and the Beatrice Hunter Cancer Research Institute (BHCRI). A.R. was supported by funds from the Department of Surgery, the Killam Trusts and the BHCRI Cancer Research Training Program. E.L.C. was supported by funds from the Killam Trusts, Natural Sciences and Engineering and Research Council (NSERC) and a Nova Scotia Graduate Scholarship. The authors would like to thank Garry Beattie, in memoriam, and Lori Duggan for their generosity, and support of brain cancer research through the Beattie Strong Brain Cancer Bash and QE2 Foundation. Additionally the authors would like to thank Liam Rappoldt for his experimental support.

\section{Author details}

'Department of Surgery, Dalhousie University, Halifax, NS, Canada. 2Department of Medical Neuroscience, Dalhousie University, Halifax, NS, Canada. ${ }^{3}$ Division of Neurosurgery, Department of Surgery, Dalhousie University, Halifax, NS, Canada. ${ }^{4}$ Department of Pathology, Dalhousie University, Halifax, NS, Canada. ${ }^{5}$ Department of Microbiology \& Immunology, Dalhousie University, Halifax, NS, Canada. ${ }^{6}$ Department of Biochemistry \& Molecular Biology, University of British Columbia, Vancouver, BC, Canada. ${ }^{7}$ Department of Medicine, Dalhousie University, Halifax, NS, Canada.

${ }^{8}$ Department of Microbiology, Immunology and Infectious Disease, Cumming School of Medicine, University of Calgary, Calgary, AB, Canada

\section{Conflict of interest}

The authors declare no conflict of interest.

\section{Publisher's note}

Springer Nature remains neutral with regard to jurisdictional claims in published maps and institutional affiliations.

Supplementary Information accompanies this paper at (https://doi.org/ 10.1038/s41419-020-03159-5).

Received: 12 November 2019 Revised: 9 October 2020 Accepted: 12 October 2020

Published online: 17 November 2020

\section{References}

1. Stupp, R. et al. Radiotherapy plus concomitant and adjuvant temozolomide for glioblastoma. N. Engl. J. Med. https://doi.org/10.1056/NEJMoa043330 (2005).

2. Silvera, D., Formenti, S. C. \& Schneider, R. J. Translational control in cancer. Nat. Rev. Cancer https://doi.org/10.1038/nrc2824 (2010).

3. Ruggero, D. Translational control in cancer etiology. Cold Spring Harb. Perspect. Biol. https://doi.org/10.1101/cshperspect.a012336 (2013).

4. Anderson, P., Kedersha, N. \& Ivanov, P. Stress granules, P-bodies and cancer. Biochim. Biophys. Acta-Gene Regul. Mech. https:/doi.org/10.1016/j. bbagrm.2014.11.009 (2015).

5. El-Naggar, A. M. \& Sorensen, P. H. Translational control of aberrant stress responses as a hallmark of cancer. J. Pathol. https://doi.org/10.1002/path.5030 (2018).

6. Shibao, S. et al. Metabolic heterogeneity and plasticity of glioma stem cells in a mouse glioblastoma model. Neuro-Oncology https://doi.org/10.1093/ neuonc/nox170 (2018).

7. Helmy, K. et al. Identification of global alteration of translational regulation in glioma in vivo. PLOS ONE https://doi.org/10.1371/journal. pone.0046965 (2012). 
8. Lupinacci, F. C. S. et al. Polysome profiling of a human glioblastoma reveals intratumoral heterogeneity. Int. J. Mol. Sci. https://doi.org/10.3390/ ijms20092177 (2019).

9. Pakos-Zebrucka, K. et al. The integrated stress response. EMBO Rep. https://doi. org/10.15252/embr.201642195 (2016).

10. Anderson, P. \& Kedersha, N. Stress granules: the Tao of RNA triage. Trends Biochem. Sci. https://doi.org/10.1016/j.tibs.2007.12.003 (2008).

11. Protter, D. S. W. \& Parker, R. Principles and properties of stress granules. Trends Cell Biol. https://doi.org/10.1016/j.tcb.2016.05.004 (2016).

12. Arimoto, K., Fukuda, H., Imajoh-Ohmi, S., Saito, H. \& Takekawa, M. Formation of stress granules inhibits apoptosis by suppressing stress-responsive MAPK pathways. Nat. Cell Biol. https://doi.org/10.1038/ncb1791 (2008).

13. Dalton, L. E., Healey, E., Irving, J. \& Marciniak, S. J. Phosphoproteins in stressinduced disease. Prog. Mol. Biol. Transl. Sci. https://doi.org/10.1016/B978-0-12396456-4.00003-1 (2012)

14. Ganassi, M. et al. A surveillance function of the HSPB8-BAG3-HSP70 chaperone complex ensures stress granule integrity and dynamism. Mol. Cell https://doi. org/10.1016/j.molcel.2016.07.021 (2016).

15. Mateju, D. et al. An aberrant phase transition of stress granules triggered by misfolded protein and prevented by chaperone function. EMBO J. https://doi. org/10.15252/embj.201695957 (2017).

16. Alberti, S., Mateju, D., Mediani, L. \& Carra, S. Granulostasis: protein quality control of RNP granules. Front. Mol. Neurosci. https:/doi.org/10.3389/ fnmol.2017.00084 (2017).

17. Koumenis, C. et al. Regulation of protein synthesis by hypoxia via activation of the endoplasmic reticulum kinase PERK and phosphorylation of the translation initiation factor elF2. Mol. Cell. Biol. https://doi.org/10.1128/ mcb.22.21.7405-7416.2002 (2002).

18. Papadakis, A. I. et al. elF2a kinase PKR modulates the hypoxic response by stat3-dependent transcriptional suppression of HIF-1a. Cancer Res. https:/doi. org/10.1158/0008-5472.CAN-10-0215 (2010).

19. Liu, $Y$. et al. Regulation of $\mathrm{G} 1$ arrest and apoptosis in hypoxia by PERK and GCN2-mediated elF2a phosphorylation. Neoplasia https:/doi.org/10.1593/ neo.91354 (2010).

20. Morita, T., Satoh, R., Umeda, N., Kita, A. \& Sugiura, R. The stress granule protein $\mathrm{Vg} \mid 1$ and poly(A)-binding protein Pab1 are required for doxorubicin resistance in the fission yeast Schizosaccharomyces pombe. Biochem. Biophys. Res. Commun. https://doi.org/10.1016/j.bbrc.2011.11.127 (2012)

21. Kaehler, C., Isensee, J., Hucho, T., Lehrach, H. \& Krobitsch, S. 5-Fluorouracil affects assembly of stress granules based on RNA incorporation. Nucleic Acids Res. https://doi.org/10.1093/nar/gku264 (2014).

22. Adjibade, P. et al. Sorafenib, a multikinase inhibitor, induces formation of stress granules in hepatocarcinoma cells. Oncotarget https://doi.org/10.18632/ oncotarget.5980 (2015).

23. Szaflarski, W. et al. Vinca alkaloid drugs promote stress-induced translational repression and stress granule formation. Oncotarget https://doi.org/10.18632/ oncotarget.8728 (2016).

24. Dimasi, P., Quintiero, A., Shelkovnikova, T. A. \& Buchman, V. L. Modulation of pelF2a cellular levels and stress granule assembly/disassembly by trehalose. Sci. Rep. https://doi.org/10.1038/srep44088 (2017).

25. Bowman, R. L., Wang, Q., Carro, A. Verhaak, R. G. W. \& Squatrito, M. GlioVis data portal for visualization and analysis of brain tumor expression datasets. NeuroOncology https://doi.org/10.1093/neuonc/now247 (2017).

26. Simon, R., Mirlacher, M. \& Sauter, G. Immunohistochemical analysis of tissue microarrays. Methods Mol. Biol. https://doi.org/10.1007/978-1-60761-806-5_12 (2010).

27. Balgi, A. D. et al. Screen for chemical modulators of autophagy reveals novel therapeutic inhibitors of mTORC1 signaling. PLOS ONE https://doi.org/10.1371/ journal.pone.0007124 (2009).

28. Carpenter, A. E. et al. CellProfiler: Image analysis software for identifying and quantifying cell phenotypes. Genome Biol. https://doi.org/10.1186/gb-2006-710-r100 (2006).

29. Kedersha, N. et al. G3BP-Caprin1-USP10 complexes mediate stress granule condensation and associate with $40 \mathrm{~S}$ subunits. J. Cell Biol. https:/doi.org/ 10.1083/jcb.201508028 (2016)

30. Tsaytler, P., Harding, H. P., Ron, D. \& Bertolotti, A. Selective inhibition of a regulatory subunit of protein phosphatase 1 restores proteostasis. Science (80.). https://doi.org/10.1126/science.1201396 (2011).

31. Hui, A. M. et al. Agents with selective estrogen receptor (ER) modulator activity induce apoptosis in vitro and in vivo in ER-negative glioma cells. Cancer Res. https://doi.org/10.1158/0008-5472.CAN-04-2740 (2004).
32. Lin, S. L. et al. ER-a36, a variant of ER-a, promotes tamoxifen agonist action in endometrial cancer cells via the MAPK/ERK and PI3K/Akt pathways. PLOS ONE https://doi.org/10.1371/journal.pone.0009013 (2010).

33. Holcik, M. \& Sonenberg, N. Translational control in stress and apoptosis. Nat. Rev. Mol. Cell Biol. https://doi.org/10.1038/nrm1618 (2005).

34. Aulas, A. et al. Stress-specific differences in assembly and composition of stress granules and related foci. J. Cell Sci. https://doi.org/10.1242/jcs.199240 (2017).

35. Saxton, R. A. \& Sabatini, D. M. mTOR signaling in growth, metabolism, and disease. Cell https://doi.org/10.1016/j.cell.2017.02.004 (2017).

36. Duzgun, Z., Eroglu, Z. \& Biray Avci, C. Role of mTOR in glioblastoma. Gene https://doi.org/10.1016/j.gene.2015.08.060 (2016).

37. Weeks, A. et al. Epithelial cell transforming 2 and aurora kinase B modulate formation of stress granule-containing transcripts from diverse cellular pathways in astrocytoma cells. Am. J. Pathol. https://doi.org/10.1016/j. ajpath.2016.02.013 (2016).

38. Buchan, J. R., Kolaitis, R. M., Taylor, J. P. \& Parker, R. XEukaryotic stress granules are cleared by autophagy and Cdc48NCP function. Cell https://doi.org/ 10.1016/j.cell.2013.05.037 (2013)

39. Mauthe, M. et al. Chloroquine inhibits autophagic flux by decreasing autophagosome-lysosome fusion. Autophagy https://doi.org/10.1080/ 15548627.2018.1474314 (2018).

40. Guarino, V. A., Oldham, W. M., Loscalzo, J. \& Zhang, Y. Y. Reaction rate of pyruvate and hydrogen peroxide: assessing antioxidant capacity of pyruvate under biological conditions. Sci. Rep. https://doi.org/10.1038/s41598-01955951-9 (2019).

41. Roudier, E., Bachelet, C. \& Perrin, A. Pyruvate reduces DNA damage during hypoxia and after reoxygenation in hepatocellular carcinoma cells. FEBS $\mathrm{J}$. https://doi.org/10.1111/j.1742-4658.2007.06044.x (2007).

42. Gal, J. et al. The acetylation of lysine-376 of G3BP1 regulates RNA binding and stress granule dynamics. Mol. Cell. Biol. https://doi.org/10.1128/mcb.00052-19 (2019).

43. Matsuki, H. et al. Both G3BP1 and G3BP2 contribute to stress granule formation. Genes to Cells https://doi.org/10.1111/gtc.12023 (2013).

44. Atlas, R., Behar, L., Elliott, E. \& Ginzburg, I. The insulin-like growth factor mRNA binding-protein IMP-1 and the Ras-regulatory protein G3BP associate with tau mRNA and HuD protein in differentiated P19 neuronal cells. J. Neurochem. https://doi.org/10.1111/j.1471-4159.2004.02371.x (2004).

45. Ishii, A. et al. Histological characterization of the tumorigenic 'peri-necrotic niche' harboring quiescent stem-like tumor cells in glioblastoma. PLOS ONE https://doi.org/10.1371/journal.pone.0147366 (2016).

46. Ruggieri, A. et al. Dynamic oscillation of translation and stress granule formation mark the cellular response to virus infection. Cell Host Microbe https:// doi.org/10.1016/j.chom.2012.05.013 (2012).

47. Ghosh, S. \& Geahlen, R. L. Stress granules modulate SYK to cause microglial cel dysfunction in Alzheimer's disease. EBioMedicine https://doi.org/10.1016/j. ebiom.2015.09.053 (2015).

48. Mackenzie, I. R. et al. TIA1 Mutations in amyotrophic lateral sclerosis and frontotemporal dementia promote phase separation and alter stress granule dynamics. Neuron https://doi.org/10.1016/j.neuron.2017.07.025 (2017).

49. Wolozin, B. Regulated protein aggregation: stress granules and neurodegeneration. Mol. Neurodegeneration https://doi.org/10.1186/1750-1326-7-56 (2012).

50. Jawhari, S., Ratinaud, M. H. \& Verdier, M. Glioblastoma, hypoxia and autophagy: a survival-prone 'ménage-à-trois'. Cell Death Dis. https://doi.org/10.1038/ codis.2016.318 (2016).

51. Colwell, N. et al. Hypoxia in the glioblastoma microenvironment: Shaping the phenotype of cancer stem-like cells. Neuro-Oncology https://doi.org/10.1093/ neuonc/now258 (2017).

52. Graham, K. \& Unger, E. Overcoming tumor hypoxia as a barrier to radiotherapy, chemotherapy and immunotherapy in cancer treatment. Int. J. Nanomed. https://doi.org/10.2147/IJN.S140462 (2018).

53. Mastronardi, L., Puzzilli, F., Couldwell, W. T., Farah, J. O. \& Lunardi, P. Tamoxifen and carboplatin combinational treatment of high-grade gliomas. Results of a clinical trial on newly diagnosed patients. J. Neurooncol. https://doi.org/ 10.1023/A:1005968724240 (1998).

54. Parney, I. F. \& Chang, S. M. Current chemotherapy for glioblastoma. Cancer J. https://doi.org/10.1097/00130404-200305000-00003 (2003).

55. Kedersha, N. L., Gupta, M., Li, W., Miller, I. \& Anderson, P. RNA-binding proteins TIA-1 and TIAR link the phosphorylation of elF-2a to the assembly of mammalian stress granules. J. Cell Biol. https://doi.org/10.1083/jcb.147.7.1431 (1999).

56. Thedieck, K. et al. Inhibition of mTORC1 by astrin and stress granules prevents apoptosis in cancer cells. Cell https://doi.org/10.1016/j.cell.2013.07.031 (2013). 
57. McCormick, C. \& Khaperskyy, D. A. Translation inhibition and stress granules in the antiviral immune response. Nat. Rev. Immunol. https:/doi.org/10.1038/ nri.2017.63 (2017).

58. Buchan, J. R. \& Parker, R. Eukaryotic stress granules: the ins and outs of translation. Mol. Cell https://doi.org/10.1016/j.molcel.2009.11.020 (2009).

59. Ikediobi, O. N. et al. Mutation analysis of 24 known cancer genes in the NCl-60 cell line set. Mol. Cancer Ther. https://doi.org/10.1158/1535-7163.MCT-06-0433 (2006).

60. Feng, X. et al. Hypoxia-induced acetylation of PAK1 enhances autophagy and promotes brain tumorigenesis via phosphorylating ATG5. Autophagy https:// doi.org/10.1080/15548627.2020.1731266 (2020)

61. Abdul Rahim, S. A. et al. Regulation of hypoxia-induced autophagy in glioblastoma involves ATG9A. Br. J. Cancer https:/doi.org/10.1038/bjc.2017.263 (2017).

62. Yazğan, B., Yazğan, Y., Övey, I. S. \& Nazıroğlu, M. Raloxifene and tamoxifen reduce PARP activity, cytokine and oxidative stress levels in the brain and blood of ovariectomized rats. J. Mol. Neurosci. https://doi.org/10.1007/s12031016-0785-9 (2016)
63. Gaignard, P. et al. Role of sex hormones on brain mitochondrial function, with special reference to aging and neurodegenerative diseases. Front. Aging Neurosci. https://doi.org/10.3389/fnagi.2017.00406 (2017).

64. Geng, Y., Kohli, L., Klocke, B. J. \& Roth, K. A. Chloroquine-induced autophagic vacuole accumulation and cell death in glioma cells is p53 independent. Neuro-Oncology https://doi.org/10.1093/neuonc/nop048 (2010).

65. Button, R. W., Roberts, S. L., Willis, T. L., Oliver Hanemann, C. \& Luo, S. Accumulation of autophagosomes confers cytotoxicity. J. Biol. Chem. https://doi. org/10.1074/jbc.M117.782276 (2017).

66. Gammoh, N. et al. Role of autophagy in histone deacetylase inhibitor-induced apoptotic and nonapoptotic cell death. Proc. Natl Acad. Sci. USA https://doi. org/10.1073/pnas.1204429109 (2012).

67. Lee, J. Y. et al. HDAC6 controls autophagosome maturation essential for ubiquitin-selective quality-control autophagy. EMBO J. https://doi.org/10.1038/ emboj.2009.405 (2010)

68. Fournier, M. J., Gareau, C. \& Mazroui, R. The chemotherapeutic agent bortezomib induces the formation of stress granules. Cancer Cell Int. https://doi.org/ 10.1186/1475-2867-10-12 (2010). 